\title{
变轴线生物融合式膝关节康复机构型综合*
}

\author{
王 森 ${ }^{1,2}$ 李艳文 ${ }^{1,2}$ 陈子明 ${ }^{1,2}$ 宋加涛 $^{1,2}$ 梁文龙 $^{1,2}$ 朱为国 ${ }^{3}$ \\ (1. 燕山大学河北省并联机器人与机电系统实验室 秦皇岛 066004 ;
}

2. 燕山大学机械工程学院 秦皇岛 066004;

3. 淮阴工学院先进制造技术重点实验室 淮安 223003)

\begin{abstract}
摘要: 以一种具有变轴线转动的 $1 \mathrm{R}$ 人体膝关节模型为基础, 提出一种膝关节与外骨骼轴线自动对准的生物融合式膝关节康 复机构型综合的方法, 并综合出了一系列生物融合式膝关节康复机构。给出一种膝关节与外骨骼关节轴线自动对准的运动补 偿条件, 基于螺旋理论构建符合运动补偿条件的生物融合式膝关节康复机构的基本模型; 以生物融合式膝关节康复机构为研 究对象, 用改进的 G-K 公式分析了生物融合式膝关节康复机构的自由度, 并用运动学仿真软件验证了运动补偿的正确性, 进 而确定了机构综合的合理性, 为生物融合式膝关节康复机构型综合的设计提供了新的思路。
\end{abstract}

关键词：生物融合; 膝关节康复；螺旋理论；运动补偿；型综合；康复机构

中图分类号: TP242

\section{Type Synthesis of Rehabilitation Mechanism of Variable Axis Bio-fusion Knee Joint}

\author{
WANG Sen ${ }^{1,2}$ LI Yanwen ${ }^{1,2}$ CHEN Ziming ${ }^{1,2}$ SONG Jiatao ${ }^{1,2}$ \\ LIANG Wenlong $^{1,2}$ ZHU Weiguo ${ }^{3}$
}

(1. Parallel Robot and Mechatronic System Laboratory of Hebei Province, Yanshan University,

Qinhuangdao 066004;

2. School of Mechanical Engineering, Yanshan University, Qinhuangdao 066004;

3. Advanced Manufacturing Technology Key Laboratory, Huaiyin Institute of Technology, Huaian 223003)

\begin{abstract}
A new 1R human knee model which is based on a variable axis rotation, the method of configuration synthesis of bio-fusion knee rehabilitation machanism with automatic alignment of knee joint and exoskeleton axis are proposed, then a series of bio-fusion knee rehabilitation mechanisms are summarized. There is an effective way that a motion compensatary condition for the automatic alignment of the axis of knee joint and exoskeleton joint, and constructs the basic model of the bio-fusion knee joint rehabilitation mechanism, which is based on the spiral theory. Treating the bio-fusion knee joint rehabilitation mechanism as the research object, the freedom of the bio-fusion knee joint rehabilitation mechanism is analyzed with the improved G-K formula, and the kinematics simulation software is used the accuracy of motion compensation is verified by the simulation results, and then the rationality of mechanism synthesis is determined, which provides a new idea for the design method of configuration synthesis of bio-fusion knee joint rehabilitation machanism.
\end{abstract}

Key words: bio-fusion; rehabilitation of keen joint; spiral theory; motion compensation; type synthesis; rehabilitation mechanism

\section{0 前言}

现有的膝关节康复机构基本可分为两种类型：

* 国家自然科学基金(51775474)、河北省自然科学基金(E2016203321)、 江苏省重点实验室项目 (HGAMTL-1708) 和河北省重点研发计划 (19221909D)资助项目。20191112 收到初稿, 20200213 收到修改稿
串联机构和并联机构。具有代表性的串联机构如 CPM 训练器 ${ }^{[1]}$, 其机械结构原理上为曲柄滑块机构, 通过滑块的往复运动带动人体小腿进行膝关节的康 复运动。在实际康复过程中, 会出现膝关节和外骨 骼轴线发生相对位移，使得膝关节轴线与外骨骼轴 线之间不能共线，而且在两者绑定的位置处会产生 额外的力和力矩, 虽然两者间的相对运动被肌肉等 
软组织形变所补偿, 但是作用力和作用力矩会在康 复过程产生不良的影响, 甚至对病人造成二次伤害。 为改进这一缺点北京航空航天大学 ${ }^{[2]}$ 用平面并联机 构模拟膝关节工作空间的复杂运动, 但即使了解了 膝关节的运动轨迹, 通过运动学反解计算出驱动函 数, 由于个体差异的原因, 每个人的膝关节运动轨 迹都不尽相同, 一套康复装置无法满足所有人的康 复需求。因此, 需要研发出一种能够满足不同个体 需求的变轴线生物融合式膝关节康复机构。

为提高机构的性能, 可以以人体膝关节轴线和 外骨骼轴线相对位置的调整作为可穿戴设备设计的 目标。综上所述, 康复装置需要实现穿戴者关节与 外骨骼关节康复机构运动的一致性。可以采用型综 合的理论, 综合出一类新型的膝关节康复机构。这 类机构具有自动调整外骨骼与人体膝关节轴线相对 位置的能力，而且易于控制。

机构的型综合问题是机构学理论中一个重要的 研究内容。目前, 在少自由度并联机构型综合方面 取得的研究成果较多, 对于并联机构的型综合方法 有以下几种: HUANG 等 ${ }^{[3]}$ 提出了基于运动螺旋与 反螺旋的型综合方法, 综合出一系列分支对称的少 自由度并联机构。YANG ${ }^{[4]}$ 提出了基于单开链(SOC) 单元的并联机构型综合方法而且得到若干新型并联 机构。GOGU ${ }^{[5]}$ 采用线性变换的思想, 系统研究并 联机构自由度和型综合的问题。KONG 等 ${ }^{[6]}$ 基于虚 拟链法对几种常用运动模式的并联机构进行构型综 合。文献[7]基于 GF 集理论对仿生四足步行机器人 进行类型综合。文献[8]基于位移子群的型综合位移 流形综合原理, 对 3R2T 并联机构进行系统综合; 此外其他学者对具有连续转轴 ${ }^{[9]}$ 、螺旋运动并联机

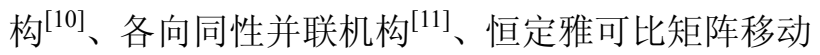

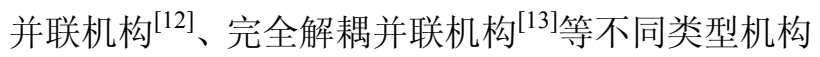
开展了型综合研究。

用螺旋理论研究空间机构的一些问题是比较方 便的。相对于其他方法, 螺旋理论 ${ }^{[14]}$ 具有几何概念 清楚、物理意义明确、表达形式简单和代数运算方 便等优点, 而且螺旋理论易于与其他方法如矢量法、 矩阵法和运动影响系数法之间相互转化。这在诸多 数学工具中是较好的一种。但是用传统螺旋理论的 型综合办法, 无法直接综合出变轴线转动的单自由 度膝关节康复机构。因此, 寻求变轴线转动的单自 由度膝关节康复机构型综合的理论和方法, 是机构 学的一个新的方向和难题。本文从具有变轴线转动 的 $1 \mathrm{R}$ 人体膝关节运动支链出发, 提出一种以膝关 节轴线自对准为前提的运动补偿条件, 构建一类生 物融合式膝关节康复新机构, 进一步丰富和扩充了
型综合的理论和方法。

\section{1 生物融合康复机构型综合方法}

由膝关节解剖学可知, 膝关节是由股骨、胫骨 和髌骨构成的复合关节, 为人体最大且构造最复杂 的关节, 如图 1 所示。膝关节的主要运动是人体在 矢状面内的屈曲运动, 并且股骨和胫骨之间的相对 运动, 是滚动和滑动的复合运动。文献[15]利用磁 共振成像(MRI)研究了膝关节的形状和相对运动, 关 节面处的运动不仅仅是围绕一个点旋转, 而且还存 在着旋转中心的移动。瞬时转动中心的轨迹可以看 成 “ $\mathrm{J}$ ” 形曲线。这项研究表明膝关节的运动不能简 化为绕固定轴旋转, 人体膝关节矢状面的运动是变 轴线的转动运动。文献[16]在 2008 年基于椭圆几何 的接触运动学理论对膝关节模型进行分析发现膝关 节的股骨关节面可以近似为椭圆。

基于上述研究, 本文提出一种轴线自对准的生 物融合式膝关节康复机构型综合的方法。首先建立 具有变轴线转动的 $1 \mathrm{R}$ 人体膝关节模型, 在此膝关 节运动分支的基础上, 通过添加串联支链的方法, 基于螺旋理论构建符合运动补偿条件的生物融合式 膝关节康复机构的基本模型; 最后以生物融合式膝 关节康复机构为研究对象, 用改进的 G-K 公式计算 了生物融合式膝关节康复机构自由度, 并用运动学 仿真软件验证了运动补偿的正确性, 从而确定了机 构综合的合理性。

\section{1 具有变轴线转动的 $1 R$ 人体膝关节三维模型}

如图 1 所示, 以具有变轴线转动的 $1 \mathrm{R}$ 人体膝关 节运动支链为研究对象, 在动定平台中间添加运动支 链构成轴线自对准的生物融合式膝关节康复机构。

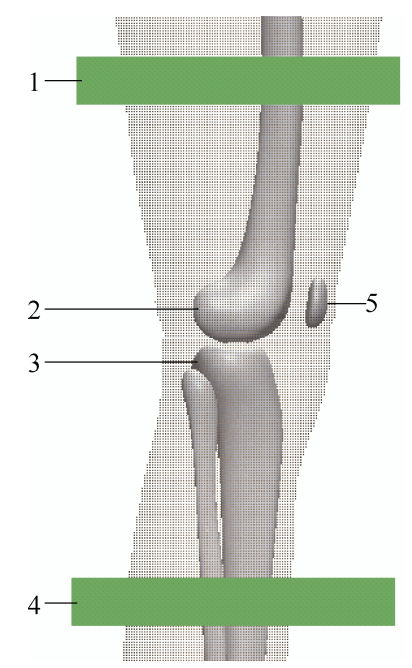

图 1 具有变轴线转动的 $1 \mathrm{R}$ 人体膝关节三维模型

1. 定平台 2 . 股骨 3 . 胫骨 4 . 动平台 5 . 髌骨 


\section{2 膝关节轴线自动对准的运动补偿原理}

如图 2 所示, 在定平台中心点建立坐标系 $o x y z$, $O$ 为坐标系原点。在动平台的基准面 $I$ 中间位置布置 驱动副, 驱动副的轴线与人体矢状面垂直。由图 2 的 几何关系可得出, 驱动副的转角 $\gamma$ 等于膝关节转动角 度 $\theta$, 可以实现完全解耦, 控制方便。并且机构中融 入人体膝关节进行分析, 实现变轴线的转动运动, 和 人体膝关节矢状面内的运动完全贴合, 自动实现运动 补偿, 图 2 中 “J” 形曲线表示膝关节瞬心变化轨迹。

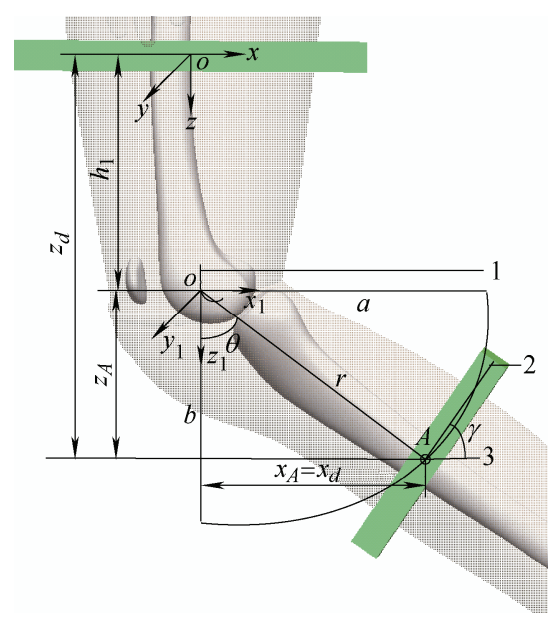

图 2 生物融合膝关节康复机构运动补偿原理图解

1. 瞬心轨迹 2. 基准面 $I$ 3. 驱动副(动平台中心点)

如图 2 分析可知, 动平台中心点的运动轨迹和 膝关节的股骨关节面一样, 近似为椭圆轨迹, 椭圆 的圆心为 $o_{1}$, 以 $o_{1}$ 为坐标系原点, 建立分支坐标系 $o_{1}-x_{1} y_{1} z_{1}, x_{1}$ 轴方向与 $x$ 轴方向平行, 定坐标系原点 $o$ 与 $o_{1}$ 在同一条直线上, 由几何关系可得

$$
\begin{aligned}
& \frac{x_{A}^{2}}{a^{2}}+\frac{z_{A}^{2}}{b^{2}}=1 \\
& x_{A}=r \sin \theta \\
& z_{A}=r \cos \theta
\end{aligned}
$$

求解式(1) (3)可得, 固定坐标系下, 动平台中 心点的位置坐标 $x_{d} 、 z_{d}$ 可以表示为

$$
\begin{gathered}
x_{d}=\frac{a b \sin \theta}{\sqrt{a^{2} \cos ^{2} \theta+b^{2} \sin ^{2} \theta}} \\
z_{d}=\frac{a b \cos \theta}{\sqrt{a^{2} \cos ^{2} \theta+b^{2} \sin ^{2} \theta}}+h_{1}
\end{gathered}
$$

式中, $h_{1}$ 为股骨关节面中心到定平台中心点沿 $z$ 轴 方向的距离; $a 、 b$ 分别表示以 $o_{1}$ 为椭圆圆心的动平 台中心点陏圆运动轨迹的长轴和短轴半径; $r$ 表示 椭圆半径; $x_{A} 、 z_{A}$ 分别表示动平台中心点 $A$ 在分支 坐标系 $o_{1} x_{1} y_{1} z_{1}$ 下的投影坐标。

通过式(4)、(5)分析可知动平台中心点的运动轨迹 由驱动副转角和人体膝关节面的形状决定, 通过控制 驱动副可以完全控制膝关节的转角, 由于不同的人有
不同的膝关节面轮廓从而导致 $a 、 b$ 是不一样的，驱动 副转角决定运动轨迹角度, 具有变轴线转动的 $1 \mathrm{R}$ 人体 膝关节决定了运动轨迹尺寸形状, 在康复运动过程保 证了动平台中心点能够走出符合膝关节运动规律的椭 圆轨迹, 无需考虑外骨骼和人体膝关节轴线之间具体 的位置变化, 自动实现运动补偿, 满足康复需求。

\section{3 膝关节轴线自对准的运动补偿条件}

为了补偿膝关节在屈伸运动过程中瞬时转动中 心的位移，提出了一种膝关节康复机构轴线自对准 的运动补偿条件。

（1）所添加的运动分支与动、定平台构成的并 联机构具有平行于膝关节矢状面的两平移一转动三 个自由度, 其中转动自由度的转轴平行于 $y$ 轴。

(2) 驱动副轴线与人体矢状面垂直, 位于动平 台的基准面 $I$ 的中心位置。

运动补偿条件(1)保证了没有确定运动的受损 膝关节在矢状面内进行正确的康复运动。

运动补偿条件(2)满足图 2 的几何条件, 这样的 布置保证了驱动转角等于膝关节转角, 结合生物融 合式膝关节康复机构运动补偿原理, 实现膝关节轴 线的自动对准。

\section{4 生物融合式膝关节康复机构型综合的步骤}

具有变轴线转动的生物融合式膝关节康复机构 其构型综合流程图如图 3 所示。

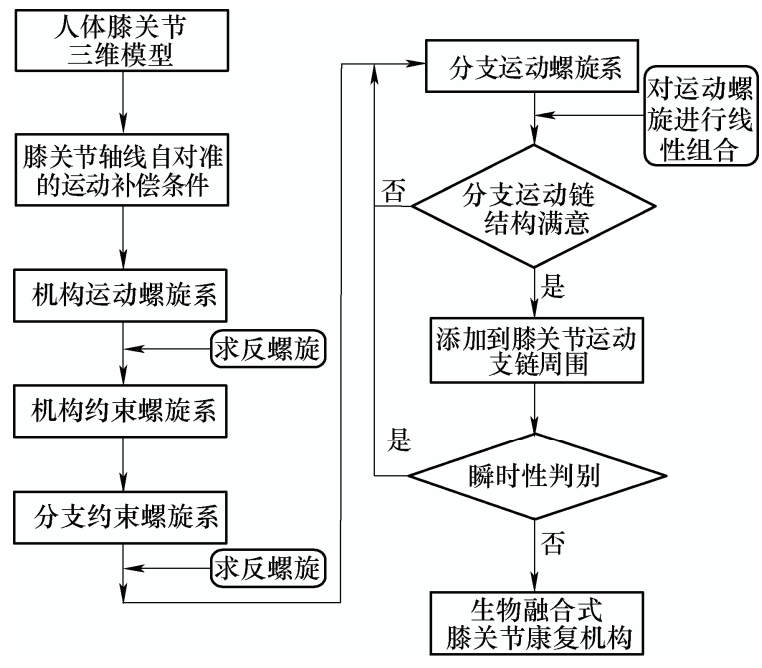

图 3 生物融合式膝关节康复机构型综合流程图

\section{2 生物融合式膝关节康复机构型综合}

首先根据运动补偿条件(1)确定所添加的串联 支链构成的并联机构所需自由度, 写出其运动螺旋 系, 通过求反螺旋的方法获得约束螺旋系, 再确定分 支约束螺旋系, 并求与分支约束螺旋系相逆的基础运 动螺旋系, 对基础运动螺旋系进行线性组合, 含有驱 
动副的分支必须按照运动补偿条件(2)构造串联运动 分支。构造出符合约束要求的串联运动分支, 根据实 际要求选择几组适当的运动分支, 添加到 $1 \mathrm{R}$ 人体膝 关节运动支链两侧, 并对机构的瞬时性进行判别, 构 造出轴线自对准的生物融合式膝关节康复机构。

\section{1 运动分支是 5 自由度的膝关节康复机构}

根据运动补偿条件(1), 需要添加的运动支链与动定 平台构成的并联机构具有平行于膝关节矢状面的两平 移一转动三个自由度, 写出满足上述条件的运动螺旋

$$
\left\{\begin{array}{l}
\$_{x}=\left(\begin{array}{llllll}
0 & 0 & 0 ; & 1 & 0 & 0
\end{array}\right) \\
\$_{z}=\left(\begin{array}{llllll}
0 & 0 & 0 ; & 0 & 0 & 1
\end{array}\right) \\
\$_{y}=\left(\begin{array}{llllll}
0 & 1 & 0 ; & 0 & 0 & 0
\end{array}\right)
\end{array}\right.
$$

对式(6)求解约束反螺旋, 得到机构的 3 个约束力螺 旋的基本解析式

$$
\left\{\begin{array}{l}
\$_{1}^{r}=\left(\begin{array}{llllll}
0 & 0 & 0 ; & 0 & 0 & 1
\end{array}\right) \\
\$_{2}^{r}=\left(\begin{array}{llllll}
0 & 0 & 0 ; & 1 & 0 & 0
\end{array}\right) \\
\$_{3}^{r}=\left(\begin{array}{llllll}
0 & 1 & 0 ; & 0 & 0 & 0
\end{array}\right)
\end{array}\right.
$$

式中的 $\boldsymbol{\$}_{1}^{r}, \quad \boldsymbol{\$}_{2}^{r}$ 表示 2 个空间相互垂直的力偶矢量, $\$_{3}^{r}$ 表示一个力线矢。
考虑分支自由度是 5 自由度的情况, 并且分支 构成的并联机构不存在公共约束, 这样并联机构中 仅含有三个分支, 而且约束力螺旋是平均分配的, 即每个分支都分别与式(7)中的一个约束力螺旋互 逆。与 $\boldsymbol{\$}_{1}^{r}$ 互逆的运动螺旋为

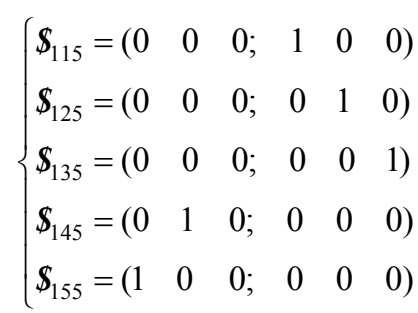

式中, $\$_{i l \mathrm{k}}$ 表示运动螺旋 $(i=1,2,3$ 表示分支序号, $l=1 ， 2 ， 3 ， 4 ， 5$ 表示运动副序号， $k=3 ， 4 ， 5$ 表示 分支自由度数)。

对式(8)中的 5 个运动螺旋进行线性组合, 线性 组合后可得到其他多种形式含驱动副的运动螺旋, 必须满足线性组合后的运动螺旋线性无关, 只有按 照运动补偿条件(2)对运动螺旋 $\$_{145} 、 \$_{115} 、 \$_{135}$ 进行 线性组合才能构成驱动副, 则根据要求可以添加的

\begin{tabular}{|c|c|c|c|c|}
\hline 分支中含两个移动副 & 分支中含一个移动副 & 分支中不含移动副 & 分支中含有圆柱副 & 分支中含有万向铰 \\
\hline${ }^{x} \mathrm{R}^{x} \mathrm{R}^{k_{1}} \mathrm{P}^{k_{2}} \mathrm{P}^{y} \mathrm{R}$ & ${ }^{k} \mathrm{P}^{x} \mathrm{R}^{x} \mathrm{R}^{y} \mathrm{R}^{y} \mathrm{R}$ & ${ }^{x} \mathrm{R}^{x} \mathrm{R}^{x} \mathrm{R}^{y} \mathrm{R}^{y} \mathrm{R}$ & ${ }^{x} \mathrm{C}^{x} \mathrm{R}^{x} \mathrm{R}^{y} \mathrm{R}$ & ${ }^{x} \mathrm{R}^{x} \mathrm{R}^{x} \mathrm{U}^{y}{ }^{y} \mathrm{R}$ \\
\hline${ }^{x} \mathrm{R}^{k_{1}} \mathrm{P}^{k_{2}} \mathrm{P}^{x} \mathrm{R}^{y} \mathrm{R}$ & ${ }^{x} \mathrm{R}^{k} \mathrm{P}^{x} \mathrm{R}^{y} \mathrm{R}^{y} \mathrm{R}$ & & ${ }^{x} \mathrm{C}^{k} \mathrm{P}^{x} \mathrm{R}^{y} \mathrm{R}$ & ${ }^{x} \mathrm{R}^{x} \mathrm{R}^{x} \mathrm{R}^{x} \mathrm{U}^{y}$ \\
\hline${ }^{k_{1}} \mathrm{P}^{x} \mathrm{R}^{x} \mathrm{R}^{k_{2}} \mathrm{P}^{y} \mathrm{R}$ & ${ }^{x} \mathrm{R}^{x} \mathrm{R}^{k} \mathrm{P}^{y} \mathrm{R}^{y} \mathrm{R}$ & & ${ }^{x} \mathrm{C}^{x} \mathrm{R}^{k} \mathrm{P}^{y} \mathrm{R}$ & ${ }^{x} \mathrm{R}^{x} \mathrm{U}^{y}{ }^{y} \mathrm{R}^{y} \mathrm{R}$ \\
\hline${ }^{k_{1}} \mathrm{P}^{k_{2}} \mathrm{P}^{x} \mathrm{R}^{x} \mathrm{R}^{y} \mathrm{R}$ & ${ }^{x} \mathrm{R}^{x} \mathrm{R}^{y} \mathrm{R}^{k} \mathrm{P}^{y} \mathrm{R}$ & & ${ }^{x} \mathrm{R}^{x} \mathrm{C}^{x} \mathrm{R}^{y} \mathrm{R}$ & ${ }^{k_{1}} \mathrm{P}^{x} \mathrm{R}^{k_{2}} \mathrm{P}^{x} \mathrm{U}^{y}$ \\
\hline${ }^{k_{1}} \mathrm{P}^{x} \mathrm{R}^{k_{2}} \mathrm{P}^{x} \mathrm{R}^{y} \mathrm{R}$ & ${ }^{x} \mathrm{R}^{k} \mathrm{P}^{x} \mathrm{R}^{x} \mathrm{R}^{y} \mathrm{R}$ & & ${ }^{x} \mathrm{R}^{x} \mathrm{R}^{x} \mathrm{C}^{y} \mathrm{R}$ & ${ }^{x} \mathrm{R}^{k} \mathrm{P}^{x} \mathrm{U}^{y y} \mathrm{R}$ \\
\hline \multirow[t]{4}{*}{${ }^{k_{1}} \mathrm{P}^{x} \mathrm{R}^{k_{2}} \mathrm{P}^{x} \mathrm{R}^{y} \mathrm{R}$} & ${ }^{x} \mathrm{R}^{x} \mathrm{R}^{k} \mathrm{P}^{x} \mathrm{R}^{y} \mathrm{R}$ & & ${ }^{k} \mathrm{P}^{x} \mathrm{C}^{x} \mathrm{R}^{y} \mathrm{R}$ & ${ }^{y} \mathrm{U}^{x x} \mathrm{R}^{x} \mathrm{U}^{y}$ \\
\hline & ${ }^{x} \mathrm{R}^{x} \mathrm{R}^{x} \mathrm{R}^{k} \mathrm{P}^{y} \mathrm{R}$ & & ${ }^{x} \mathrm{R}^{x} \mathrm{C}^{k} \mathrm{P}^{y} \mathrm{R}$ & ${ }^{y} \mathrm{U}^{x k} \mathrm{P}^{x} \mathrm{R}^{y} \mathrm{R}$ \\
\hline & & & ${ }^{k} \mathrm{P}^{x} \mathrm{R}^{x} \mathrm{C}^{y} \mathrm{R}$ & ${ }^{k} \mathrm{P}^{x} \mathrm{R}^{x} \mathrm{R}^{x} \mathrm{U}^{y}$ \\
\hline & & & & ${ }^{x} \mathrm{R}^{k} \mathrm{P}^{x} \mathrm{R}^{x} \mathrm{U}^{y}$ \\
\hline
\end{tabular}
$3 \mathrm{~T} 2 \mathrm{R}$ 运动分支支链如表 1 所示。

表 1 含有驱动副的 3T2R 运动支链

表 1 中 $\mathrm{R}$ 表示转动副, $\mathrm{P}$ 表示移动副, $\mathrm{U}$ 表示 胡克铰, $\mathrm{C}$ 表示圆柱副。字母上标 $y$ 表示运动副轴 线与图 2 的基准面 $I$ 垂直, $x$ 表示运动副轴线平行于 图 2 的固定坐标系 $x$ 轴, $k 、 k_{1} 、 k_{2}$ 表示移动副轴线 为空间任意方向。与 $\$_{2}^{r}$ 互逆的运动螺旋系表示的也 是一个被约束转动运动的螺旋系, 分析结果与式(8) 相同, 把表 1 中 $x$ 向转动副替换成 $z$ 向转动副即可, 下文符号表示同上。与 $\boldsymbol{\$}_{3}^{r}$ 互逆的运动螺旋只需要用 式(9)的 $\$ 315$ 替换式(8)的 $\$_{125}$ 即可

$$
\$_{315}=\left(\begin{array}{llllll}
0 & 0 & 1 ; & 0 & 0 & 0
\end{array}\right)
$$

通过对 $\$_{115} 、 \$_{315} 、 \$_{135} 、 \$_{145}$ 和 $\$_{155} 5$ 个运动螺旋的 线性组合, 可得到多种形式的运动螺旋, 必须满足 线性组合后的运动螺旋线性无关, 则根据要求可以 添加的 3R2T 运动分支支链如表 2 所示。

\begin{tabular}{|c|c|c|}
\hline 分支中含两个移动副 & 分支中含一个移动副 & 分支中不含移动副 \\
\hline${ }^{x z} \mathrm{P}^{x z} \mathrm{PS}$ & ${ }^{x z} \mathrm{P}^{y} \mathrm{RS}$ & ${ }^{y} \mathrm{R}{ }^{y} \mathrm{RS}$ \\
\hline $\mathrm{S}^{x z} \mathrm{P}^{x z} \mathrm{P}$ & ${ }^{y} \mathrm{R}^{x z} \mathrm{PS}$ & ${ }^{y} \mathrm{RS}^{y} \mathrm{R}$ \\
\hline${ }^{x z} \mathrm{PS}^{x z} \mathrm{P}$ & ${ }^{y} \mathrm{RS}^{x z} \mathrm{P}$ & $\mathrm{S}^{y} \mathrm{R}^{y} \mathrm{R}$ \\
\hline${ }^{x z} \mathrm{P}^{x z} \mathrm{P}^{y} \mathrm{R}(\mathrm{RR}){ }_{N}$ & ${ }^{x z} \mathrm{PS}^{y} \mathrm{R}$ & $(\mathrm{RR})_{N}{ }^{y} \mathrm{R}^{y} \mathrm{R}^{y} \mathrm{R}$ \\
\hline${ }^{x z} \mathrm{P}^{y} \mathrm{R}^{x z} \mathrm{P}(\mathrm{RR})_{N}$ & $\mathrm{~S}^{x z} \mathrm{P}^{y} \mathrm{R}$ & ${ }^{y} \mathrm{R}(\mathrm{RR})_{N}{ }^{y} \mathrm{R}^{y} \mathrm{R}$ \\
\hline${ }^{y} \mathrm{R}^{x z} \mathrm{P}^{x z} \mathrm{P}(\mathrm{RR})_{N}$ & $\mathrm{~S}^{y} \mathrm{R}^{x z} \mathrm{P}$ & ${ }^{y} \mathrm{R}{ }^{y} \mathrm{R}(\mathrm{RR})_{N}{ }^{y} \mathrm{R}$ \\
\hline${ }^{x z} \mathrm{P}^{x z} \mathrm{P}(\mathrm{RR}){ }_{N}{ }^{y} \mathrm{R}$ & ${ }^{x z} \mathrm{P}^{y} \mathrm{R}^{y} \mathrm{R}(\mathrm{RR})_{N}$ & ${ }^{y} \mathrm{R}^{y} \mathrm{R}^{y} \mathrm{R}(\mathrm{RR})_{N}$ \\
\hline${ }^{x z} \mathrm{P}^{y} \mathrm{R}(\mathrm{RR}){ }_{N}{ }^{x z} \mathrm{P}$ & ${ }^{y} \mathrm{R}^{y} \mathrm{R}^{x z} \mathrm{P}(\mathrm{RR})_{N}$ & \\
\hline${ }^{y} \mathrm{R}{ }^{x z} \mathrm{P}(\mathrm{RR}){ }_{N}{ }^{x z} \mathrm{P}$ & ${ }^{y} \mathrm{R}^{x z} \mathrm{P}^{y} \mathrm{R}(\mathrm{RR})_{N}$ & \\
\hline${ }^{x z} \mathrm{P}(\mathrm{RR}){ }_{N}{ }^{x z} \mathrm{P}^{y} \mathrm{R}$ & ${ }^{y} \mathrm{R}{ }^{x z} \mathrm{P}(\mathrm{RR}){ }_{N}{ }^{y} \mathrm{R}$ & \\
\hline${ }^{y} \mathrm{R}(\mathrm{RR})_{N}{ }^{x z} \mathrm{P}^{x z} \mathrm{P}$ & ${ }^{x z} \mathrm{P}^{y} \mathrm{R}(\mathrm{RR})_{N}{ }^{y} \mathrm{R}$ & \\
\hline${ }^{x z} \mathrm{P}(\mathrm{RR})_{N}{ }^{y} \mathrm{R}^{x z} \mathrm{P}$ & ${ }^{y} \mathrm{R}{ }^{y} \mathrm{R}(\mathrm{RR})_{N}{ }^{x z} \mathrm{P}$ & \\
\hline$(\mathrm{RR})_{N}{ }^{x z} \mathrm{P}^{x z} \mathrm{P}^{y} \mathrm{R}$ & ${ }^{x z} \mathrm{P}(\mathrm{RR})_{N}{ }^{y} \mathrm{R}{ }^{y} \mathrm{R}$ & \\
\hline$(\mathrm{RR})_{N}{ }^{x z} \mathrm{P}^{y} \mathrm{R}^{x z} \mathrm{P}$ & ${ }^{y} \mathrm{R}(\mathrm{RR}){ }_{N}{ }^{x z} \mathrm{P}^{y} \mathrm{R}$ & \\
\hline \multirow[t]{3}{*}{$(\mathrm{RR})_{N}{ }^{y} \mathrm{R}^{x z} \mathrm{P}^{x z} \mathrm{P}$} & $(\mathrm{RR})_{N}{ }^{x z} \mathrm{P}^{y} \mathrm{R}^{y} \mathrm{R}$ & \\
\hline & ${ }^{y} \mathrm{R}(\mathrm{RR}){ }_{N}{ }^{y} \mathrm{R}{ }^{x z} \mathrm{P}$ & \\
\hline & $(\mathrm{RR})_{N}{ }^{y} \mathrm{R}^{y} \mathrm{R}^{x z} \mathrm{P}$ & \\
\hline
\end{tabular}

其中 $(\mathrm{RR})_{N}$ 表示 $2 \mathrm{R}$ 球面子副, 下标 $N$ 表示连续的 两个转动副轴线交于同一点, 该点称为分支中心点。
表 23 R2T 运动分支支链 
根据上述综合出的 5 自由度的串联分支添加到 $1 \mathrm{R}$ 人体膝关节运动支链周围, 构造出能自对准膝关节轴线 的生物融合式康复机构。如图 4、5 中仅列出了两种 5 自由度分支结构简单的机型。 $\mathrm{R}_{\mathrm{K}}$ 表示膝关节运动支链。

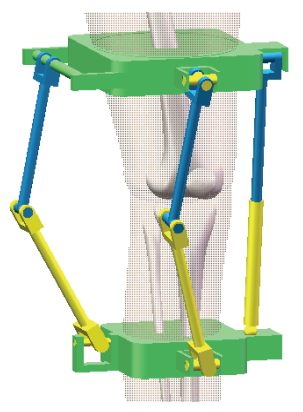

图 $4{ }^{x} \mathrm{C}^{x} \mathrm{R}^{x} \mathrm{U}^{y} p^{y} \mathrm{U}^{x x} \mathrm{R}^{x} \mathrm{U}^{y}{ }^{y} \mathrm{R}^{x z} \mathrm{PS} / \mathrm{R}_{\mathrm{K}}$ 生物融合膝关节康复机构

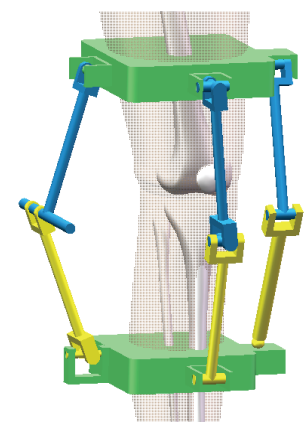

图 $5{ }^{x} \mathrm{R}^{x} \mathrm{C}^{x} \mathrm{U}^{y} y^{y} \mathrm{U}^{x x} \mathrm{U}^{y}{ }^{y} \mathrm{R} /{ }^{y} \mathrm{R}^{y} \mathrm{RS} / \mathrm{R}_{\mathrm{K}}$ 生物融合膝关节康复机构

\section{2 运动分支是 4 自由度的膝关节康复机构}

对 $\boldsymbol{\$}_{1}^{r} 、 \boldsymbol{\$}_{2}^{r} 、 \boldsymbol{\$}_{3}^{r}$ 任意两个约束螺旋求反螺旋, 即可得到两种类型的运动分支, 分别对 $\boldsymbol{\$}_{1}^{r} 、 \boldsymbol{\$}_{3}^{r}$ 和 $\boldsymbol{\$}_{2}^{r} 、 \boldsymbol{\$}_{3}^{r}$ 两组约束螺旋求逆可得到 $2 \mathrm{~T} 2 \mathrm{R}$ 运动分支, 对 $\boldsymbol{S}_{1}^{r} 、 \boldsymbol{\$}_{2}^{r}$ 求逆可得到 $3 \mathrm{~T} 1 \mathrm{R}$ 运动分支。

例如对 $\boldsymbol{\$}_{1}^{r} 、 \boldsymbol{\$}_{3}^{r}$ 求逆可得运动螺旋为

$$
\left\{\begin{array}{l}
\$_{114}=\left(\begin{array}{llllll}
0 & 0 & 0 ; & 1 & 0 & 0
\end{array}\right) \\
\$_{124}=\left(\begin{array}{llllll}
0 & 0 & 0 ; & 0 & 0 & 1
\end{array}\right) \\
\$_{134}=\left(\begin{array}{llllll}
1 & 0 & 0 ; & 0 & 0 & 0
\end{array}\right) \\
\$_{144}=\left(\begin{array}{llllll}
0 & 1 & 0 ; & 0 & 0 & 0
\end{array}\right)
\end{array}\right.
$$

通过对式(10)中的 4 个运动螺旋线性组合, 可 以得到多种形式的 $2 \mathrm{~T} 2 \mathrm{R}$ 运动螺旋系, 必须满足线 性组合后的运动螺旋线性无关, 同理可以得到 3T1R 的多个运动支链, 根据要求可以添加的 4 自由度运 动支链如表 3 所示。

表 3 四自由度的运动支链

\begin{tabular}{cc}
\hline 2T2R 运动支链 & 3T1 $\mathrm{R}$ 运动支链 \\
\hline${ }^{x} \mathrm{U}^{y}{ }^{y} \mathrm{R}^{y} \mathrm{R}$ & ${ }^{x z} \mathrm{P}^{k_{1}} \mathrm{P}^{k_{2}} \mathrm{P}^{y} \mathrm{R}$ \\
${ }^{x z} \mathrm{P}^{x} \mathrm{P}^{x} \mathrm{R}^{y} \mathrm{R}$ & ${ }^{x} \mathrm{P}^{z} \mathrm{P}^{y} \mathrm{C}$ \\
${ }^{x z} \mathrm{P}^{x} \mathrm{C}^{y} \mathrm{R}$ & ${ }^{x y} \mathrm{P}^{k} \mathrm{P}^{y} \mathrm{R}^{y} \mathrm{R}$ \\
${ }^{x} \mathrm{C}^{x z} \mathrm{P}^{y} \mathrm{R}$ & ${ }^{x y} \mathrm{P}^{y} \mathrm{R}^{k} \mathrm{P}^{y} \mathrm{R}$ \\
${ }^{x} \mathrm{C}^{y} \mathrm{R}^{y} \mathrm{R}$ & ${ }^{y} \mathrm{C}^{x} \mathrm{P}^{z} \mathrm{P}$ \\
& ${ }^{k} \mathrm{P}^{x y} \mathrm{P}^{y} \mathrm{R}{ }^{y} \mathrm{P}$ \\
& ${ }^{x z} \mathrm{P}^{y} \mathrm{C}^{y} \mathrm{R}$ \\
\hline
\end{tabular}

把表 3 中 $2 \mathrm{~T} 2 \mathrm{R}$ 运动支链里 $x$ 向转动副替换成 $z$ 向转动副, 即可得到与 $\boldsymbol{\$}_{2}^{r} 、 \$_{3}^{r}$ 互逆的 $2 \mathrm{~T} 2 \mathrm{R}$ 运动 支链。

上述综合出来的 4 自由度串联分支添加到 $1 \mathrm{R}$ 人体膝关节运动支链周围, 构造出能自对准膝关节 轴线的生物融合式康复机构, 如图 6 所示。

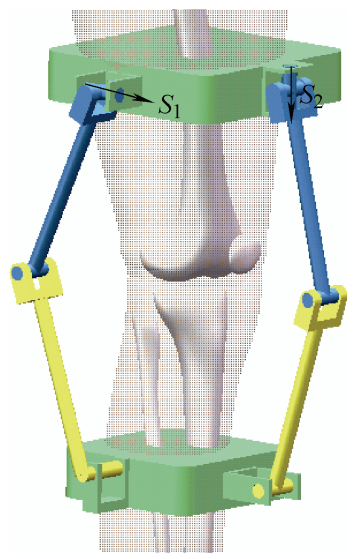

图 $6{ }^{x} \mathrm{U}^{y y} \mathrm{R}^{y} \mathrm{R} /{ }^{z} \mathrm{U}^{y}{ }^{y} \mathrm{R}^{y} \mathrm{R} / \mathrm{R}_{\mathrm{K}}$ 生物融合膝关节康复机构

通过分析可知图 6 机构在运动过程, 和定平台 固连的转动副轴线 $S_{1} 、 S_{2}$ 方向始终不变, 去掉这两 个穴余运动副, 机构同样能实现两平移一转动三个 自由度, 此时机构变成 $2 R R R / R_{K}$, 机构分支自由度 为 3 , 下文将具体介绍三自由度的运动分支, 通过 分析可知单纯的四自由度分支无法构成满足运动补 偿条件的串联分支，因为有些运动副在机构空间布局 确定后被其他分支所约束。由于上文已经构造出 5 自 由度的运动分支, 将 5 自由度和 4 自由度的运动分支 组合布置在 $1 \mathrm{R}$ 人体膝关节运动支链周围, 构造出能 自对准膝关节轴线的生物融合式康复机构。如图 7 所 示, 在定平台中心点建立直角坐标系 $o x y z$, 图 7 中的 URR 分支限制了动平台 $y$ 轴方向的移动和 $x$ 方向的转 动, $U R U$ 分支限制了 $z$ 方向的转动, URR/URU 并联 机构限制了 $y$ 轴方向的平移和 $x z$ 方向的转动, 只存在 两平移一转动三个自由度, 满足运动补偿需求。

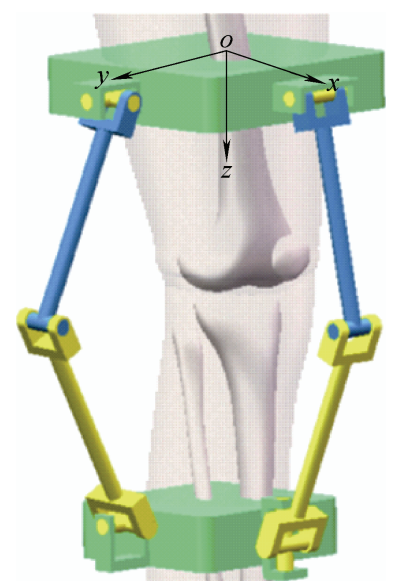

图 $7{ }^{z} \mathrm{U}^{y}{ }^{y} \mathrm{R}^{y} \mathrm{R} /{ }^{y} \mathrm{U}^{x}{ }^{x} \mathrm{R}^{x} \mathrm{U}^{y} / \mathrm{R}_{\mathrm{K}}$ 生物融合膝关节康复机构 


\section{3 运动分支是 3 自由度的膝关节康复机构}

对 $\boldsymbol{\$}_{1}^{r} 、 \$_{2}^{r}$ 和 $\boldsymbol{\$}_{3}^{r}$ 求逆可得到三自由度的运动分支

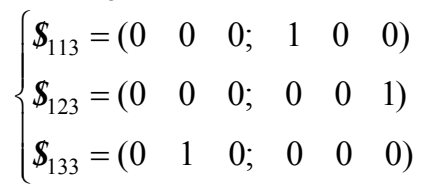

通过式(11)中 3 个运动螺旋的线性组合, 就可以得 到许多满足约束条件的运动分支, 必须满足线性组合 后的螺旋线性无关, 综合得到的 $2 \mathrm{~T} 1 \mathrm{R}$ 运动分支构型可 以表示为: ${ }^{y} \mathrm{R}^{x z} \mathrm{P}^{y} \mathrm{R} 、{ }^{y} \mathrm{R}^{y} \mathrm{R}{ }^{y} \mathrm{R} 、{ }^{x z} \mathrm{P}^{x z} \mathrm{P}^{y} \mathrm{R}$ 、 ${ }^{x z} \mathrm{P}^{y} \mathrm{R}^{y} \mathrm{R} 、{ }^{x z} \mathrm{P}^{y} \mathrm{R}{ }^{x z} \mathrm{P} 、{ }^{y} \mathrm{R}^{x z} \mathrm{P}^{x z} \mathrm{P} 、{ }^{y} \mathrm{R}^{y} \mathrm{R}^{x z} \mathrm{P}$ 。

根据上述综合出来的 3 自由度串联分支添加到 $1 \mathrm{R}$ 人体膝关节运动支链周围, 构造出能自对准膝关 节轴线的生物融合式康复机构。如图 8、9 所示。

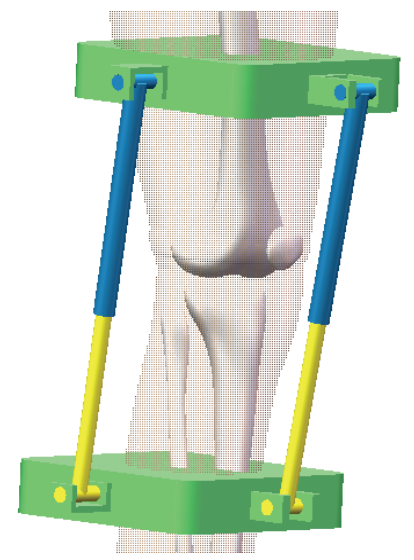

图 $8 \quad 2^{y} \mathrm{R}^{x z} \mathrm{P}^{y} \mathrm{R} / \mathrm{R}_{\mathrm{K}}$ 生物融合膝关节康复机构

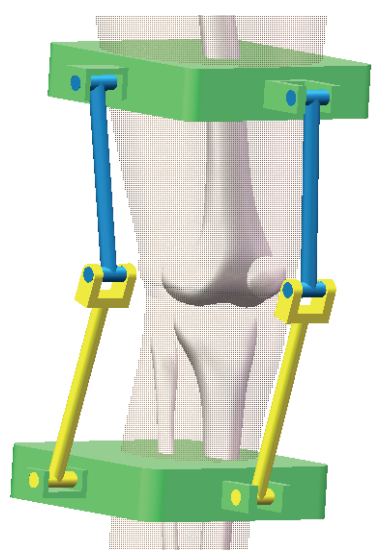

图 $92^{y} \mathrm{R}^{y} \mathrm{R}^{y} \mathrm{R} / \mathrm{R}_{\mathrm{K}}$ 生物融合膝关节康复机构

\section{$2.4 \mathrm{CRU} / \mathrm{RRR} / \mathbf{R}_{\mathrm{K}}$ 膝关节康复机构自由度分析}

以 $C R U / R R R / R_{K}$ 康复机构为例, 对本文综合的 机构进行分析, 以验证综合方法的正确性。

根据螺旋理论分析了 $\mathrm{CRU} / \mathrm{RRR} / \mathrm{R}_{\mathrm{K}}$ 康复机构自 由度, 如图 10 所示在定平台中心处建立基坐标系 $M-X Y Z, \$_{12}$ 与 $X$ 轴平行, $\$_{11}$ 与 $Y$ 轴平行, 驱动副 按照运动补偿条件(2)进行布置。CRU 分支的运动螺 旋 $\$_{12} 、 \$_{13} 、 \$_{14}$ 相互平行, RRR 分支转动副方向相
互平行, 膝关节处运动副轴线方向沿着 $Y$ 轴方向。

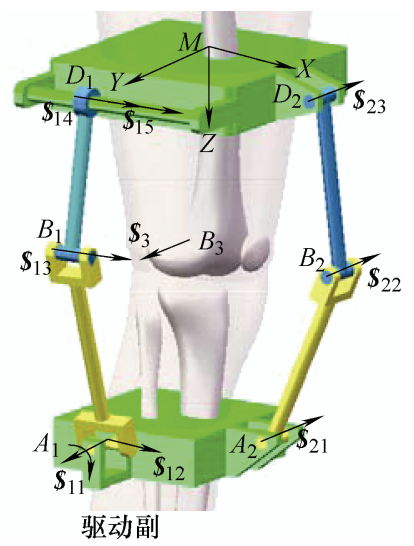

图 $10 \mathrm{CRU} / \mathrm{RRR} / \mathrm{R}_{\mathrm{K}}$ 膝关节康复机构

按照图 10 所示的运动螺旋分布图, 可以写出 $\mathrm{CRU} / \mathrm{RRR} / \mathrm{R}_{\mathrm{K}}$ 康复机构三个分支运动副在基坐标系 $M-X Y Z$ 中的运动螺旋, 利用螺旋互易积计算反螺旋 的方法, 可以求得各个分支的约束反螺旋。

$\mathrm{CRU}$ 分支运动螺旋系为

$$
\left\{\begin{array}{l}
\$_{11}=\left(\begin{array}{llllll}
0 & 1 & 0 ; & -Z_{A 1} & 0 & X_{A 1}
\end{array}\right) \\
\$_{12}=\left(\begin{array}{llllll}
1 & 0 & 0 ; & 0 & Z_{A 1} & -Y_{A 1}
\end{array}\right) \\
\$_{13}=\left(\begin{array}{llllll}
1 & 0 & 0 ; & 0 & -Z_{B 1} & Y_{B 1}
\end{array}\right) \\
\$_{14}=\left(\begin{array}{llllll}
1 & 0 & 0 ; & 0 & 0 & -Y_{D 1}
\end{array}\right) \\
\$_{15}=\left(\begin{array}{llllll}
0 & 0 & 0 ; & 1 & 0 & 0
\end{array}\right)
\end{array}\right.
$$

CRU 分支约束螺旋系为

$$
\boldsymbol{\$}^{r}=\left(\begin{array}{llllll}
0 & 0 & 0 ; & 0 & 0 & 1
\end{array}\right)
$$

$R R R$ 分支运动螺旋系如下

$$
\left\{\begin{array}{l}
\$_{21}=\left(\begin{array}{llllll}
0 & 1 & 0 ; & -Z_{A 2} & 0 & X_{A 2}
\end{array}\right) \\
\$_{22}=\left(\begin{array}{llllll}
0 & 1 & 0 ; & -Z_{B 2} & 0 & X_{B 2}
\end{array}\right) \\
\$_{23}=\left(\begin{array}{llllll}
0 & 1 & 0 ; & 0 & 0 & X_{D 2}
\end{array}\right)
\end{array}\right.
$$

$R R R$ 分支反螺旋系为

$$
\left\{\begin{array}{l}
\$_{21}^{r}=\left(\begin{array}{llllll}
0 & 0 & 0 ; & 1 & 0 & 0
\end{array}\right) \\
\boldsymbol{\$}_{22}^{r}=\left(\begin{array}{llllll}
0 & 0 & 0 ; & 0 & 0 & 1
\end{array}\right) \\
\$_{23}^{r}=\left(\begin{array}{llllll}
0 & 1 & 0 ; & 0 & 0 & 0
\end{array}\right)
\end{array}\right.
$$

膝关节分支运动螺旋系为

$$
\$_{3}=\left(\begin{array}{llllll}
0 & 1 & 0 ; & -Z_{B 3} & 0 & X_{B 3}
\end{array}\right)
$$

膝关节分支约束螺旋系为

$$
\left\{\begin{array}{l}
\boldsymbol{\$}_{31}^{r}=\left(\begin{array}{llllll}
0 & 1 & 0 ; & 0 & 0 & 0
\end{array}\right) \\
\boldsymbol{\$}_{32}^{r}=\left(\begin{array}{llllll}
0 & 0 & 0 ; & 1 & 0 & 0
\end{array}\right) \\
\boldsymbol{\$}_{33}^{r}=\left(\begin{array}{llllll}
0 & 0 & 0 ; & 0 & 0 & 1
\end{array}\right) \\
\boldsymbol{\$}_{34}^{r}=\left(\begin{array}{llllll}
1 & 0 & 0 ; & 0 & Z_{B 3} & 0
\end{array}\right) \\
\boldsymbol{\$}_{35}^{r}=\left(\begin{array}{llllll}
0 & 0 & 1 ; & 0 & -X_{B 3} & 0
\end{array}\right)
\end{array}\right.
$$


$\$_{i j}$ 表示此机构运动螺旋 $(i=1,2,3$ 表示分支序 号, $j=1 ， 2 ， 3 ， 4 ， 5$ 表示运动副序号)， $X_{m k} 、 Y_{m k}$ 、 $Z_{m k}(m=A, B, D$ 表示运动副中心点代号, $k=1,2$, 3 表示分支序号)表示各分支运动副中心点的位置。

不考虑公共约束的情况下机构的阶数取 6 ,

CRU 分支、 RRR 分支和膝关节分支共受到 9 个约束 螺旋, 这三个运动分支受到的约束螺旋的最大线性 无关数是 5 , 整个机构存在 4 个元余约束。

用修正的 G-K 公式计算机构的自由度

$$
\begin{gathered}
M=6(n-g-1)+\sum_{i=1}^{g} f_{i}+v= \\
6(6-7-1)+9+4=1
\end{gathered}
$$

式中 $M-$ 一机构的自由度;

$n$ 一包括机架构件的数目;

$g$ 一一运动副的数目;

$f_{i}$ 一一第 $i$ 个运动副的自由度;

$v$ 一一多环并联机构在不考虑公共约束的情 况下先余约束的数目。

可以验证膝关节康复机构的动平台存在 1 个绕 着 $Y$ 轴方向转动的自由度。

动平台自由度与人体膝关节自由度相同, 如式 (16)所示, 始终表示一个沿着人体矢状面法线方向 转动, 位置不断变化的单自由度运动, 因此该机构 是一个全周自由度机构。

\subsection{CRU $/ R R R / R_{K}$ 康复机构仿真验证}

为了验证型综合出来的生物融合式膝关节康复 机构运动补偿的正确性, 用仿真软件对康复机构进 行运动仿真, 如图 11 所示, 在定平台中心点建立直 角坐标系 $O X Y Z, x_{2} 、 z_{2}$ 分别表示动平台中心点与定 坐标系原点 $O$ 之间距离在 $X 、 Z$ 方向的投影, 同理, $x_{4} 、 z_{4}$ 分别表示另一组股骨关节面尺寸不同的膝关 节模型的动平台中心点与定坐标系原点 $O$ 之间距离 在 $X 、 Z$ 方向的投影, $\theta$ 表示膝关节的转角。

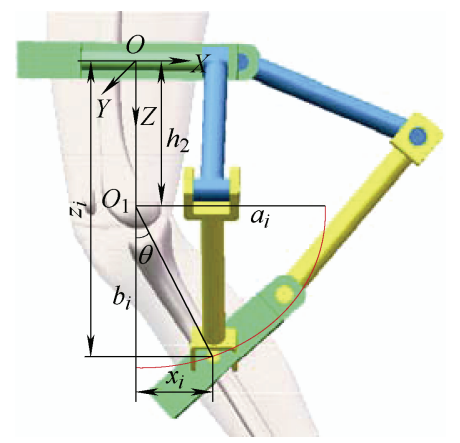

图 11 康复机构运动仿真模型

在仿真过程中康复机构对两组不同尺寸的人体 膝关节模型在 0 到 $60^{\circ}$ 的运动过程中任意取 6 个角
度, 测量动平台中心点的位置坐标, 与理论计算的结 果进行比较, 理论值采用式(4)、(5)计算出动平台中心 点的位置坐标。如表 4、5 所示, $x_{1} 、 z_{1}$ 和 $x_{3} 、 z_{3}$ 分 别表示不同尺寸的人体膝关节模型计算的理论值, 按照人机工程学设置理论模型结构参数 $a_{1}=244.98 \mathrm{~mm}, a_{2}=246.62 \mathrm{~mm}, b_{1}=233.98 \mathrm{~mm}$, $b_{2}=236.02 \mathrm{~mm}, h_{1}=177 \mathrm{~mm}$ 。两种算例仿真时机构 的结构参数 $b_{3}=233.98 \mathrm{~mm}, b_{4}=236.02 \mathrm{~mm}$, $h_{2}=177 \mathrm{~mm}, h(i=1,2)$ 分别表示理论和仿真模型中股 骨关节面中心到定平台中心点的距离, $a_{i} 、 b_{i}(i=1,2)$ 分别表示图 2 中不同尺寸的膝关节理论模型中以 $o_{1}$ 为椭圆圆心的动平台中心点走出的椭圆运动轨迹的 长轴和短轴半径, $b_{i}(i=3,4)$ 分别表示运动算例 $1 、 2$ 仿真过程中动平台与定平台平行时, 股骨关节面中 心到动平台中心点的距离。

表 4 运动算例 1 验证

\begin{tabular}{cccccc}
\hline 膝关节转角 & \multicolumn{2}{c}{ 理论计算 $/ \mathrm{mm}$} & & \multicolumn{2}{c}{ 仿真算例 $/ \mathrm{mm}$} \\
\cline { 2 - 3 } \cline { 5 - 6 }$\left({ }^{\circ}\right)$ & $x_{1}$ & $z_{1}$ & & $x_{2}$ & $z_{2}$ \\
\hline 10 & 40.68 & 407.72 & & 40.68 & 407.72 \\
20.07 & 80.70 & 397.88 & & 80.70 & 397.88 \\
30.6 & 120.44 & 380.65 & & 120.44 & 380.65 \\
44.8 & 168.45 & 346.63 & & 168.45 & 346.63 \\
53 & 192.1 & 321.78 & & 192.1 & 321.78 \\
60 & 209.40 & 297.90 & & 209.40 & 297.90 \\
\hline
\end{tabular}

表 5 运动算例 2 验证

\begin{tabular}{cccccc}
\hline \multirow{2}{*}{$\begin{array}{c}\text { 膝关节转角 } \\
\theta /(\stackrel{\circ}{ })\end{array}$} & \multicolumn{2}{c}{ 理论计算 $/ \mathrm{mm}$} & & \multicolumn{2}{c}{ 仿真算例 $/ \mathrm{mm}$} \\
\cline { 2 - 3 } \cline { 5 - 6 } & $x_{3}$ & $z_{3}$ & & $x_{4}$ & $z_{4}$ \\
\hline 10 & 41.04 & 409.73 & & 41.04 & 409.73 \\
20.07 & 81.39 & 399.79 & & 81.39 & 399.79 \\
30.6 & 121.48 & 382.40 & & 121.48 & 382.40 \\
44.8 & 169.89 & 348.08 & & 169.89 & 348.08 \\
53 & 193.76 & 323.01 & & 193.76 & 323.01 \\
60 & 211.17 & 298.92 & & 211.17 & 298.92 \\
\hline
\end{tabular}

通过表 4、5 两组数据分别对比发现, 理论值和 仿真值完全一致, 验证了膝关节康复机构综合的合 理性。

\section{3 结论}

(1) 建立了具有变轴线转动 $1 R$ 人体膝关节三 维模型, 以此 $1 \mathrm{R}$ 膝关节模型为基础, 提出一种膝 关节轴线与外骨骼轴线自动对准的运动补偿条件和 一类生物融合式膝关节康复机构型综合的新方法。

（2）基于螺旋理论构建符合运动补偿条件的生物 融合式膝关节康复机构的基本模型; 最后以一种生物 
融合式膝关节康复机构为研究对象, 用修正的 G-K 公 式计算了生物融合式膝关节康复机构自由度, 并用运 动学仿真软件验证了运动补偿的正确性, 为进一步综 合生物融合式膝关节康复机构提供新的思路。

\section{参 考 文 献}

[1] ZHU Lili, WANG Guangxin, LI Dan. Design of CPM training machine of recovery for knee joints[J]. Applied Mechanics and Materials, 2013, 2567(734): 161-164.

[2] LYU Mingxing, CHEN Weihai, DING Xilun, et al. Design of a biologically inspired lower limb exoskeleton for human gait rehabilitation[J]. The Review of Scientific Instruments, 2016, 87(10): 289-304.

[3] HUANG Zhen, LI Qinchuan. Type synthesis of symmetrical lower-mobility parallel mechanisms using the constraint-synthesis method[J]. International Journal of Robotics Research, 2003， 22(1): 59-79.

[4] SHEN Huiping, YANG Tingli, MA Lyuzhong. Synthesis and structure analysis of kinematic structures of 6-DOF parallel robotic mechanisms[J]. Mechanism and Machine Theory, 2004, 40(10): 1164-1180.

[5] GOGU G, Structural synthesis of fully-isotropic translational parallel robots via theory of linear transformations[J]. European Journal of Mechanics / A Solids, 2004, 23(6): 1021-1039.

[6] KONG Xianwen, JIN Yan. Type synthesis of 3-DOF multi-mode translational/spherical parallel mechanisms with lockable joints[J]. Mechanism and Machine Theory, 2016, 96(2): 323-333.

[7] HE Jun, GAO Feng. Type synthesis for bionic quadruped walking robots[J]. Journal of Bionic Engineering, 2015, 12(4): $527-538$.

[8] LI Qinchuan, HUANG Zhen, HERVE J M. Type synthesis of 3R2T 5-DOF parallel mechanisms using the lie group of displacements[J]. IEEE Transactions on Robotics and Automation: A Publication of The IEEE Robotics and Automation Society, 2004, 20(2): 173-180.

[9] 李仕华, 田志立, 王子义, 等. 具有连续转轴的对称 2R1T 三自由度并联机构型综合 $[\mathrm{J}]$. 机械工程学报, 2017, 53(23): 74-82.

LI Shihua, TIAN Zhili, WANG Ziyi, et al. Synthesis of symmetric 2R1T three-degree-of-freedom parallel mechanism with continuous rotation axis[J]. Journal of Mechanical Engineering, 2017, 53(23): 74-82.

[10] 杨彦东, 甄春江, 侯雨雷, 等. 对称单自由度螺旋运动 并联机构型综合[J]. 机械工程学报, 2019, 55(3): 27-33.
YANG Yandong, QI Chunjiang, HOU Yulei, et al. Synthesis of symmetric single degree of freedom spiral motion parallel mechanism[J]. Journal of Mechanical Engineering, 2019, 55(3): 27-33.

[11] 张彦斌, 刘宏昭, 吴金䥓于互易螺旋理论的无奇异完 全各向同性移动并联机构型综合 $[\mathrm{J}]$. 机械工程学报, 2008, 44(10): 83-88.

ZHANG Yanbin, LIU Hongzhao, WU Xin. Synthesis of singularly complete isotropic mobile parallel mechanism based on reciprocal spiral theory[J]. Chinese Journal of Mechanical Engineering, 2008，44(10): 83-88.

[12] 赵延治, 曹亚超, 梁博文, 等. 一类恒定雅可比矩阵移 动并联机构的判定与综合 [J]. 机械工程学报, 2017, 53(5): 101-107.

ZHAO Yanzhi, CAO Yachao, LIANG Bowen, et al. Judgement and synthesis of a class of constant jacobian matrix moving parallel mechanisms[J]. Journal of Mechanical Engineering, 2017, 53(5): 101-107.

[13] ZENG Daxing, HUANG Zhen. Type synthesis of the rotational decoupled parallel mechanism based on screw theory[J]. Science China Technological Sciences, 2011, 54(4): 998-1004.

[14] 黄真, 赵永生, 赵铁石. 高等空间机构学 $[\mathrm{M}]$. 北京: 高等教育出版社, 2006.

HUANG Zhen, ZHAO Yongsheng, ZHAO Tieshi. Advanced spatital mechanism[M]. Beijing : Higher Education Press, 2006.

[15] IWAKI $\mathrm{H}$, PINSKEROVA $\mathrm{V}$, FREEMAN M A. Tibiofemoral movement 1: The shapes and relative movements of the femur and tibia in the unloaded cadaver knee[J]. Journal of Bone \& Joint Surgery-british Volume, 2000, 82(8): 1189-1195.

[16] LEE K M, GUO Jiajie. Kinematic and dynamic analysis of an anatomically based knee joint[J]. Journal of Biomechanics, 2010, 43(7): 1231-1236.

作者简介: 王森, 男, 1995 年出生。主要研究方向为膝关节康复机器人 的设计分析。

E-mail: 2743824384@qq.com

李艳文(通信作者), 女, 1966 年出生, 博士, 教授。主要研究方向为机 器人技术及应用研究。

E-mail: ywl@ysu.edu.cn

陈子明, 男, 1984 年出生, 博士, 副教授。主要研究方向为空间少自由 度并联机构的设计与分析理论。

E-mail: chenzm@ysu.edu.cn

宋加涛, 男, 1996 年出生, 硕士研究生。主要研究方向为串联机械臂的 设计与分析。

E-mail: 2814438520@qq.com

梁文龙, 男, 1993 年出生, 硕士研究生。主要研究方向为下肢康复机器 人的设计分析。

E-mail: 2831984347@qq.com 